\title{
BCG Vaccine Induced Tuberculous Osteomyelitis of Left Distal Femur in a Young Child: Case Report and Literature Review
}

Lin $\mathrm{LC}^{1}$, Sy $\mathrm{LB}^{1 *}$, Chang $\mathrm{J} \mathrm{H}^{2}$ and Sun $\mathrm{LC}^{1}$

${ }^{1}$ Department of Pediatrics, Cardinal Tien Hospital, New

Taipei City, Taiwan

2Department of Orthopedics, Cardinal Tien Hospital, New

Taipei City, Taiwan

*Corresponding author: Leticia Sy, Department of

Pediatrics, Cardinal Tien Hospital, New Taipei City,

Taiwan

Received: May 15, 2021; Accepted: May 31, 2021;

Published: J une 07, 2021

\section{Introduction}

Tuberculosis (TB) is an essential problem for healthcare systems especially in developing countries. TB continues to pose a significant global health burden [1]. Bacillus Calmette-Guérin (BCG) is an important vaccine used to prevent Tuberculosis (TB), especially meningeal TB and disseminated TB disease in children [2]. BCG is prepared from live bovine tuberculosis bacillus, and is given to protect against TB. Although vaccination against TB by means of BCG is widespread all over the world and is generally considered to be safe, but serious adverse reactions can occur. These may be minor such as abscess formation or skin ulceration at the site of vaccination to major adverse reaction such as fatal disseminated infection especially in patients with immune deficiency [3]. These adverse reactions after BCG vaccination depend on the BCG dose, vaccine strain, vaccine administration method, injection technique, and recipient's underlying immune status [4].

The aim of this case report is to point out that clinical suspicion of BCG-induced osteomyelitis is warranted in pediatric patients with chronic symptoms of pain, limping, swelling and a limited ROM in the extremity. We also aimed to remind that imaging and culture studies may guide the clinician although tissue biopsies and genetic tests can confirm the histopathology and to review related articles.

\section{Case Presentation}

A 1 year 9 months old boy was brought to our pediatric OPD for a progressively enlarging and painful left knee mass for more than one month. History of injury to said knee with swelling for more than one month ago was informed. BCG vaccination was received at 5 months of age. There were no symptoms of fever, chronic cough, any weight lost, lethargy nor poor appetite noted. Ultrasonographic examination of medial portion of left knee with linear array transducer was performed and found; 1. one hypoechoic soft tissue mass $4.0 \times 3.6$ x $1.8 \mathrm{~cm}$ with floating multiple hyperechoic foci noted over medial aspect of left knee, 2. discontinuity of left lower femur suspicious of fracture, 3. hypoechoic and hyperechoic foci in the medullary region of left lower femur suspicious of tumor with pathologic fracture and granulation tissue. Tentative diagnosis by sonographic examination was pathologic fracture of left lower femur with granulation tissue over medial aspect of left knee. Radiographic study showed lytic lesions at distal femoral metadiaphysis, with periosteal reaction and soft tissue swelling (Figure 1). Laboratory tests showed leukocytosis with normal C-reactive protein. MRI of the left knee without contrast enhancement showed; 1 . a $4.2 \mathrm{~cm}$ multiloculated cystic lesion with extraosseous and epiphyseal extension in medial aspect of distal femoral metaphysis (Figure 2), 2. cortical erosion and bony sclerosis, 3. intact anterior and posterior cruciate ligaments and intact medial and fibular collateral ligaments, 4 . no evidence of meniscal tear or joint effusion or chondromalacia patellae. Operative management by debridement and saucerization, curettage of the distal femur and left long leg splint were done. Acid Fast (AFB) stain and TB culture of the bony tissue specimen were performed and were also sent to CDC. Results revealed the etiology of osteomyelitis was due to Mycobacterium bovis, which was proved to be BCG related. No TB contact history noted. Histopathology of bony tissue specimen revealed chronic granulomatous inflammation with foci of necrosis and multinucleated giant cells suggestive of Langhans' giant cells; no fungal or mycobacterial microorganisms are seen on PAS, GMS and

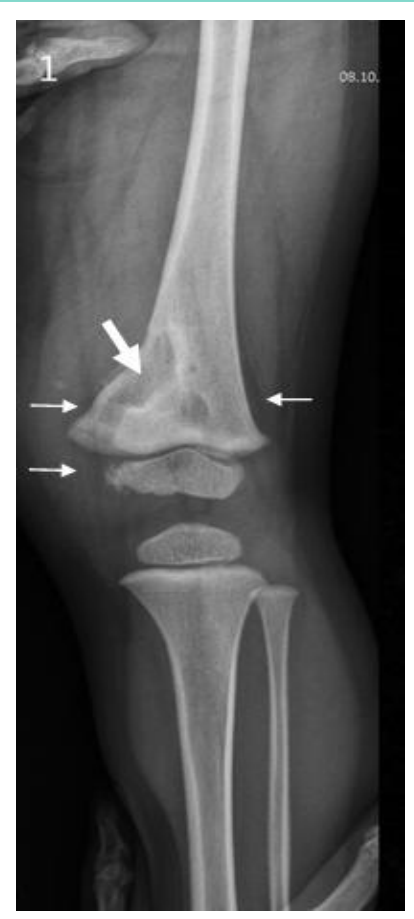

Figure 1: Osteolytic lesion at distal femoral metadiaphysis (thick arrow), with periosteal reaction and soft tissue swelling (thin arrows). 


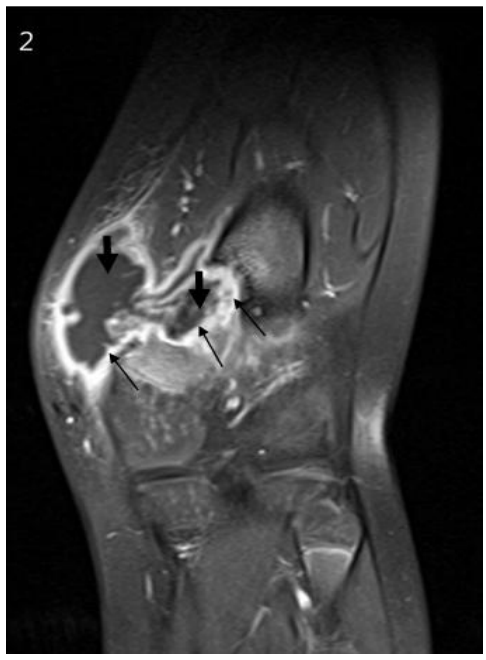

Figure 2: A 4.2cm multiloculated cystic lesion (thick arrows) with extraosseous (left arrow) and epiphyseal (right arrow) extension in medial aspect of distal femoral metaphysis; cortical erosion and bony sclerosis (thin arrows) are present.

AFB stained sections in our hospital. Early morning gastric aspirate was also collected for 3 consecutive mornings for AFB stain and TB cultures. The gastric aspirate tests were negative hence the exclusion of any existing pulmonary TB. Patient's immunologic studies were normal. Then anti-TB treatment was initiated with Isoniazid + Rifampin + Pyrazinamide (first two drugs taken for 9 months).

\section{Discussion}

Osteomyelitis is a rare complication following BCG vaccination in immunocompetent hosts, and its incidence varies between countries. A meta-analysis of previous reports indicated the frequency of BCG osteomyelitis as one in 80,000 in some European countries [5]. The incidence of BCG osteitis/osteomyelitis in Taiwan was 30.1 cases per million vaccines in 2008-2012. This late complication may occur in children within a few months to a few years after the said vaccination. Taiwan Centers for Disease Control database w/ thirty-eight patients with Mycobacterium bovis BCG-associated osteomyelitis/osteitis reported symptoms or signs began 3-32 months (average $12.4 \pm$ 6.1 months) after BCG vaccination; for $68 \%$, symptoms or signs developed 7-18 months after vaccination [6].

Many authors have reported osteomyelitis and infectious arthritis may follow vaccination with BCG in children who have normal immunity as well as in those who have immune defects. How BCG vaccine leads to osteitis is not clearly understood, but hematogenous seeding is the most likely mechanism, considering the distance between the inoculation site and locations of infection. The BCG bacteria spread rapidly throughout the body if deposited in or under the skin. The bacteria could be traced to several internal organs less than one hour after their introduction [3] The incubation period for BCG osteomyelitis is usually more than 6 months after vaccination. Previous reports of BCG osteitis/osteomyelitis showed extremity bones were more commonly involved than axial bones [7]. The lesions are localized in the metaphysis or epiphysis of long bones. Children with lower extremity long bone involvement had a longer interval from receipt of BCG vaccine to presentation (median, 16.0 months), and those with foot bone infection had higher rates of swelling (94.1\%) and local tenderness (76.5\%) [8].

The BCG osteitis/osteomyelitis is generally highly susceptible to antituberculous drugs. Nevertheless, the most effective chemotherapy regimen for the treatment of this condition is not well established. Treatment for a period of 9-12 months together with surgical evacuation usually results in good outcome [9]. It is important to diagnose BCG osteitis/osteomyelitis as early as possible, since management is very effective when started at the beginning of the disease. However, there is the possibility of defects occurring in the bone and restriction of the articular movement when the diagnosis and treatment are delayed. Reports of children with vertebral and multifocal infections had major sequelae with kyphosis or leg length discrepancy. Outcomes were good for children with involvement of the ribs, sternum, and peripheral bones without multifocal involvement. The average time for functional recovery was $6.2 \pm$ 3.9 months [8]. Polymerase Chain Reaction (PCR) method is $100 \%$ sensitive and specific for the identification of BCG among strains of the Mycobacterium tuberculosis complex.

\section{Conclusion}

BCG induced osteitis/osteomyelitis, although rare, should be kept in mind when assessing a BCG vaccinated child presenting with unexplained and prolonged local swelling or tenderness of the lower extremity even without evidence of pulmonary tuberculosis or a tuberculosis contact history. Clinical suspicion, early diagnosis using tissue biopsy and PCR studies, surgical drainage, and early commencement of chemotherapy are necessary for good outcome. It is important to note that the benefits of the vaccine in terms of reducing TB-related morbidity and mortality overcome the potential risk of BCG-related complications [1].

\section{Acknowledgement}

Thanks to the patient and his family.

\section{Authors' Contributions}

Dr. Leticia B. Sy drafted the initial manuscript, Dr. Jen-Huei Chang reviewed and Dr. Li Chuan Shun revised the manuscript.

All authors contributed to acquisition of case details and the analysis and interpretation of them.

All authors approved the final manuscript as submitted and agree to be accountable for all aspects of the work.

\section{References}

1. Al-Azri MS, Manarang J, Al-Mufargi Y. Bacille Calmette-Guérin VaccineInduced Tuberculous Hip Osteomyelitis in an Infant: Case report. Sultan Qaboos Univ Med J. 2017; 17: e358-e362.

2. Colditz GA, Brewer TF, Berkey CS, Wilson ME, Burdick E, Fineberg HV, et al. Efficacy of BCG vaccine in the prevention of tuberculosis: meta-analysis of the published literature. JAMA. 1994; 271: 698-702.

3. Al-Jassir FF, Aldeeri RA, Alsiddiky AMM, Zamzam MM. Osteomyelitis following Bacille Calmette-Guérin vaccination. Saudi Med J. 2012; 33: 87-90.

4. Lotte A, Wasz-Höckert O, Poisson N, Dumitrescu N, Verron M, Couvet E. A bibliography of the complications of BCG vaccination. A comprehensive list of the world literature since the introduction of BCG up to July 1982 , supplemented by over 100 personal communications. Adv Tuberc Res. 1984; 


\section{1: 194-245}

5. Tabatabaie P, Mahjoub F, Mehdizadeh M, Tabatabaie F. Osteomyelitis due to BCG vaccination. Indian Pediatr. 2008; 45: 930-932.

6. Chiu NC, Lin MC, Lin WL, Wang SY, Chi H, Huang LM, et al. Mycobacterium bovis BCG-associated osteomyelitis/osteitis, Taiwan. Emerg Infect Dis. 2015; 21: $539-540$.

7. Koyama A, Toida I, Nakata S. Osteitis as a complication of BCG vaccination Kekkaku. 2009; 84: 125-132.
8. Huang $\mathrm{CY}$, Chiu NC, Chi H, Huang FU, Chang PH. Clinical Manifestations, Management, and Outcomes of Osteitis/ Osteomyelitis Caused by Mycobacterium bovis Bacillus Calmette-Guérin in Children: Comparison by Site(s) of Affected Bones J Pediatr. 2019; 207: 97-102.

9. Kroger L, Korppi M, Brander E, H Kröger, O Wasz-Höckert, A Backman, et al. Osteitis caused by bacille Calmette-Guerin vaccination: a retrospective analysis of 222 cases. J Infect Dis. 1995; 172: 574-576. 